\title{
MANEJO NUTRICIONAL E ENRIQUECIMENTO AMBIENTAL NA DISFUNÇÃO COGNITIVA CANINA
}

\author{
Rebeca de Sousa Meneses ${ }^{\text {a* }}$ \\ Franciele de Oliveira Costab
}

a Graduanda de Medicina Veterinária - Centro Universitário Dr. Leão Sampaio, Av. Maria Letícia Leite Pereira s/n, Lagoa Seca - Cidade Universitária, Juazeiro do Norte - CE

b Zootecnista e Professora do Curso de Medicina Veterinária - Centro Universitário Dr. Leão Sampaio, Av. Maria Letícia Leite Pereira s/n, Lagoa Seca - Cidade Universitária, Juazeiro do Norte - CE

\section{RESUMO}

A Síndrome da Disfunção Cognitiva Canina é uma doença neurocomportamental que não tem cura e acomete cães idosos com mais de 8 anos de idade. A mudança alimentar com o uso de antioxidantes como o betacaroteno, vitaminas como a E, C e B e minerais como o zinco e magnésio ajudam a reduzir a produção de espécies reativas de oxigênio e diminuir a deposição das placas beta-amiloiode, e o enriquecimento ambiental é um grande aliado para ajudar na memória e interação social. O presente trabalho teve como o objetivo com essa revisão é discutir as estratégias alimentares e de enriquecimento ambiental para prevenção e tratamento da Disfunção Cognitiva Canina. Para atingir o objetivo proposto foi realizado um tipo de estudo descritivo bibliográfi co afi $m$ de buscar opções de alimentação e enriquecimento ambiental para os cães afetados pela DCC, buscando através da literatura cientifi ca estudos atuais acerca do tema. De acordo com as pesquisas foram compilados um total de 15 artigos, esses artigos possuíam informações sobre o tratamento farmacológico com ansiolíticos, do manejo nutricional com alimentação natural e rações, e das formas de enriquecimento ambiental de diversos tipos como o alimentar e cognitivo que devem ser utilizadas para desacelerar a progressão da doença. O uso de antioxidantes e vitaminas neuroprotetoras na dieta é de suma importância pois trazem bons resultados na memória, aprendizagem e imunidade. O enriquecimento ambiental tem que ser composto de atividade física através de passeios diários por caminhos diferentes, interação social com outros animais e pessoas e estimulação cognitiva. Á vista disso a mudança alimentar juntamente com o uso do enriquecimento ambiental são alternativas para desacelerar o avanço da DCC e aumentar a qualidade de vida do cão.

Palavras-chave: Cognitivo; Canino; Neurologia

*Autor correspondente: Rebeca de Sousa Meneses; Rua Manoel Farias de Melo; (88) 988721217; rebecamenesess@gmail.com 


\section{INTRODUÇÃO}

A Síndrome da Disfunção Cognitiva Canina (DCC) é uma patologia comportamental e neurológica que afeta cães idosos com mais de 8 anos de idade (SEKSEL 2012; AZKONA. et al. 2009; LANDSBERG \& ARAUJO, 2005). Os animais afetados apresentam mudanças no seu comportamento, tais como alteração do ciclo de sono-vigília, confusão e desorientação, aumento de irritabilidade, diminuição da resposta sensorial, lentidão em responder a comandos, mudanças socioambientais e deixam de fazer suas necessidades nos locais habituais (BOSCH et al., 2012; LIMA, 2018).

Os cães, assim como os humanos, apresentam algumas mudanças no cérebro quando envelhecem como atrofia da parte cortical, degeneração mielínica na massa branca, acúmulo de proteínas degradadas, dano no DNA e redução de antioxidantes endógenos. Com isso, ocorre dano nas proteínas, lipídios e nucleotídeos que podem contribuir para a degeneração e disfunção neuronal que acontece com a idade e nas doenças neurodegenerativas (COTMAN et al., 2002).

Por se tratar de uma doença onde os primeiros sinais são de cunho comportamental, a incidência de subdiagnóstico é alta. Entretanto, outro fator imprescindível que prejudica na chegada ao diagnóstico é que os tutores muitas vezes não convivem tempo suficiente com os animais para notar logo no início dos sinais ou porque acham que a presença deles é algo natural do envelhecimento (SILVA, 2018).

O diagnóstico in vivo é feito basicamente por exclusão de outras doenças como neoplasias cerebrais, hepatopatias e doenças hormonais, com base em exames de imagem e laboratoriais (KRUG, 2016), exame neurológico e aplicação de questionário para alterações comportamentais que os tutores devem responder. O diagnóstico definitivo só pode ser dado com a avaliação neuropatológica post mortem (KRUG, 2018).

A Disfunção Cognitiva Canina não possui tratamento com função de cura, mas sim para desacelerar a evolução da doença com o uso de fármacos como a Seleginina, em conjunto com um suporte nutricional por meio de uma dieta rica em antioxidantes e neuroprotetores e enriquecimento ambiental com passeios regulares e interação social (LANDSBERG, 2005).

$O$ tratamento pode ser feito com o uso de fármacos para deixar os neurotransmissores em níveis normais, como a utilização de diazepam ou alozopram como ansiolícos, e a melatonina 30 minutos antes da hora de dormir, para acalmar o cão durante a noite (SILVA, 2018) e com a seleginina que é opção de escoIha aprovado para o uso em cães com DCC, ela tem efeito antioxidante e neuroprotetor (KRUG, 2016), isso juntamente com mudança na dieta e enriquecimento ambiental.

Alguns trabalhos como o de Silva (2018), Pereira (2016) e Krug (2016) abordam a disfunção cognitiva canina com foco em todos os aspectos da doença como os sinais clínicos, formas de diagnóstico e tratamento, porém sem uma explicação tão detalhada sobre quais ingredientes necessitam estar na dieta do animal e onde consegui-los, e quais atividades devem ser feitas para enriquecer o ambiente e melhorar a cognitividade dos cães afetados, devido a esses fatores se faz necessário uma revisão de literatura focada na alimentação e enriquecimento ambiental. Sendo assim, o objetivo com essa revisão é discutir as estratégias alimentares e de enriquecimento ambiental para prevenção e tratamento da Disfunção Cognitiva Canina.

\section{METODOLOGIA}

Para desenvolver uma pesquisa se estabelece em primeiro momento a pergunta norteadora, que direciona o pesquisador na busca por uma investigação. Este trabalho possui como principal indagação: Como as estratégias alimentares e de enriquecimento ambiental podem auxiliar na prevenção e tratamento da Disfunção Cognitiva Canina?

Para atingir o objetivo proposto foi realizado um tipo de estudo descritivo bibliográfico afim de buscar opções de alimentação e enriquecimento ambiental para os cães afetados pela DCC, buscando através da literatura cientifica estudos atuais acerca do tema citado.

Realizou-se uma pesquisa no Google Acadêmico, Pub Med, PubVet e sites eletrôni- 
cos. A coleta de dados foi realizada entre os meses de agosto e setembro do ano de 2020, utilizou-se os seguintes termos para busca "disfunção cognitiva canina", "nutrição neurológica cães", "antioxidantes", "enriquecimento ambiental cães", abrangendo as modalidades de pesquisa: relatos de caso, pesquisas experimentais e revisões de literatura, selecionado estudos entre os anos de 1999 a 2021, nos idiomas português e inglês.

Os artigos foram primeiramente selecionados com base nos títulos. Posteriormente realizou-se então a leitura dos resumos desses trabalhos, selecionando artigos que abordassem o tema proposto. Como uma das últimas etapas de compilação foi feito a leitura completa e ao final 15 artigos relevantes ao tema atendiam aos seguintes critérios de inclusão.

Em seguida foi realizado um quadro síntese com os principais trabalhos encontrados e para discussão dos resultados optou-se por discutir em tópicos de acordo com o assunto em questão.

\section{RESULTADOS E DISCUSSÃO}

De acordo com as pesquisas foram compilados um total de 15 artigos, esses artigos possuíam informações sobre o tratamento farmacológico com ansiolíticos, do manejo nutricional com alimentação natural e rações, e das formas de enriquecimento ambiental de diversos tipos como o alimentar e cognitivo que devem ser utilizadas para desacelerar a progressão da doença (Quadro 1). Para melhor discutir os achados dividiremos essa seção nos seguintes tópicos: manejo nutricional onde iremos abordar os nutrientes necessários e onde se encontram e o enriquecimento ambiental com ideias para estimulação dos cães.

\section{Quadro 1 - Artigos utilizados para compor a pesquisa}

\begin{tabular}{|c|c|c|c|c|}
\hline Título & Autores & Ano & Objetivo & Resultados \\
\hline $\begin{array}{l}\text { A Síndrome da Disfun- } \\
\text { ção Cognitiva Canina }\end{array}$ & PEREIRA & 2009 & $\begin{array}{l}\text { Explorar a A Síndrome da } \\
\text { Disfunção Cognitiva Canina } \\
\text { (SDCC), através de uma } \\
\text { revisão bibliográfica e de } \\
\text { um pequeno estudo realiza- } \\
\text { do em cães com } 8 \text { ou mais } \\
\text { anos, para analisar a preva- } \\
\text { lência de SDCC diagnostica- } \\
\text { da e também a frequência } \\
\text { relativa de alterações com- } \\
\text { portamentais compatíveis } \\
\text { com esta patologia. }\end{array}$ & $\begin{array}{l}54,9 \% \text { dos cães com } 8 \text { ou mais } \\
\text { anos evidenciava alterações } \\
\text { comportamentais em duas ou } \\
\text { mais categorias compatíveis com } \\
\text { SDCC, sendo por isso possível } \\
\text { que alguns animais da amostra } \\
\text { padeçam desta patologia, sem } \\
\text { estarem diagnosticados. }\end{array}$ \\
\hline $\begin{array}{l}\text { Radicais livres e os } \\
\text { principais antioxidan- } \\
\text { tes da dieta }\end{array}$ & $\begin{array}{l}\text { BI ANCHI; } \\
\text { ANTUNES. }\end{array}$ & 1999 & $\begin{array}{l}\text { Discutir a importância dos } \\
\text { antioxidantes da dieta sobre } \\
\text { as estratégias de defesa dos } \\
\text { organismos contra os radi- } \\
\text { cais livres }\end{array}$ & $\begin{array}{l}\text { O uso de vitaminas e outros antio- } \\
\text { xidantes na prevenção e modula- } \\
\text { ção das consequências patológi- } \\
\text { cas dos radicais livres precisa da } \\
\text { definição de doses e de protocolo } \\
\text { de tratamento, sendo necessários } \\
\text { mais estudos sobre o mecanismo } \\
\text { de ação desses agentes antes da } \\
\text { sua prescrição em larga escala. }\end{array}$ \\
\hline
\end{tabular}




\begin{tabular}{|c|c|c|c|c|}
\hline 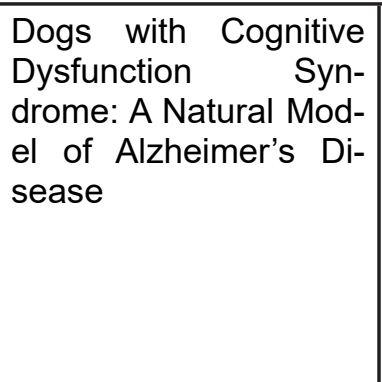 & $\begin{array}{l}\text { BOSCH et } \\
\text { al., }\end{array}$ & 2012 & $\begin{array}{l}\text { Promover novas metas para } \\
\text { o desenvolvimento de novas } \\
\text { abordagens terapêuticas } \\
\text { para a intervenção precoce } \\
\text { da DCC. }\end{array}$ & $\begin{array}{l}\text { Os cães parecem ser uma forma } \\
\text { mais simples e prática. } \\
\text { Modelo para avaliação de sujei- } \\
\text { tos geriátricos e farmacologia es- } \\
\text { tudos, eles poderiam evitar o uso } \\
\text { de primatas não humanos, e os } \\
\text { resultados mostram que a quali- } \\
\text { dade de vida de cães idosos po- } \\
\text { tencial para ser melhorado. }\end{array}$ \\
\hline $\begin{array}{l}\text { Brain aging in the ca- } \\
\text { nine: a diet enriched in } \\
\text { antioxidants reduces } \\
\text { cognitive dysfunction }\end{array}$ & $\begin{array}{l}\text { COTMAN et } \\
\text { al., }\end{array}$ & 2002 & $\begin{array}{l}\text { Avaliar os efeitos cognitivos } \\
\text { de uma dieta antioxidante } \\
\text { em caninos idosos }\end{array}$ & $\begin{array}{l}\text { A dieta resultou em uma melho- } \\
\text { ra significativa na capacidade de } \\
\text { animais idosos, mas não jovens, } \\
\text { de adquirir tarefas de aprendiza- } \\
\text { do progressivamente mais difí- } \\
\text { ceis. }\end{array}$ \\
\hline $\begin{array}{l}\text { Do dog owners rec- } \\
\text { ognise behavioural } \\
\text { indicators of canine } \\
\text { cognitive dysfunction } \\
\text { and can environmental } \\
\text { enrichment techniques } \\
\text { slow its progression? }\end{array}$ & $\begin{array}{l}\text { COUP- } \\
\text { LAND; } \\
\text { REYNOLDS }\end{array}$ & 2018 & $\begin{array}{l}\text { Determinar se os donos de } \\
\text { cães mais velhos são capa- } \\
\text { zes de perceber mudanças } \\
\text { comportamentais e, além } \\
\text { disso, explorar o conheci- } \\
\text { mento em torno do termo en- } \\
\text { riquecimento ambiental, que } \\
\text { pode ajudar os proprietários } \\
\text { a retardar a progressão do } \\
\text { DCC por meio de educação } \\
\text { adicional. }\end{array}$ & $\begin{array}{l}\text { Proprietários de cães geriátricos } \\
\text { que trabalham em uma área rela- } \\
\text { cionada à veterinária foram mais } \\
\text { propensos a notar mudanças } \\
\text { comportamentais possivelmente } \\
\text { associadas ao DCC em compara- } \\
\text { ção com o proprietário médio de } \\
\text { um cão geriátrico, e também fo- } \\
\text { ram mais propensos a entender o } \\
\text { termo enriquecimento ambiental. }\end{array}$ \\
\hline $\begin{array}{l}\text { Environmental en- } \\
\text { richment as a method } \\
\text { to improve cognitive } \\
\text { function. What can we } \\
\text { learn from animal mo- } \\
\text { dels? }\end{array}$ & FISCHER & 2016 & $\begin{array}{l}\text { Revisar os dados atualmen- } \\
\text { te disponíveis com foco es- } \\
\text { pecífico em doenças neuro- } \\
\text { degenerativas. }\end{array}$ & $\begin{array}{l}\text { Existe evidencias substanciais de } \\
\text { modelos para doenças neurode- } \\
\text { generativas que a combinação de } \\
\text { atividade física e cognitiva pode } \\
\text { melhorar a memória. }\end{array}$ \\
\hline $\begin{array}{l}\text { Canine cognitive dys- } \\
\text { function - recognition } \\
\text { and treatment }\end{array}$ & DRUCE & 2015 & $\begin{array}{l}\text { Abordar as formas de reco- } \\
\text { nhecer e tratar a disfunção } \\
\text { cognitiva canina }\end{array}$ & $\begin{array}{l}\text { É importante enfatizar aos } \\
\text { proprietários que a melhoria é } \\
\text { improvável se a modificação } \\
\text { comportamental não for realizada } \\
\text { e continuada. Isso pode ser } \\
\text { implementado juntamente com } \\
\text { a terapia medicamentosa e } \\
\text { mudanças na dieta, mas é um } \\
\text { aspecto vital e essencial do } \\
\text { tratamento. }\end{array}$ \\
\hline $\begin{array}{l}\text { Síndrome de disfunção } \\
\text { cognitiva canina }\end{array}$ & KRUG et al., & 2020 & $\begin{array}{l}\text { Serão abordados tópicos } \\
\text { como os aspectos epide- } \\
\text { miológicos, incidência da } \\
\text { doença, sinais clínicos e fi- } \\
\text { siopatologia, entre outros, } \\
\text { com objetivo de informar e } \\
\text { auxiliar no diagnóstico mais } \\
\text { preciso dessa síndrome. }\end{array}$ & $\begin{array}{l}\text { Pode-se concluir que através des- } \\
\text { sas informações é possível auxi- } \\
\text { liar no diagnóstico da síndrome } \\
\text { de disfunção cognitiva canina na } \\
\text { rotina Clínica, devido a aborda- } \\
\text { gem dos métodos de avalição do } \\
\text { paciente no consultório, questio- } \\
\text { nário, testes cognitivos, tratamen- } \\
\text { to farmacológico e suplementa- } \\
\text { ção nutricional. }\end{array}$ \\
\hline
\end{tabular}




\begin{tabular}{|c|c|c|c|c|}
\hline $\begin{array}{l}\text { Dietary supplementa- } \\
\text { tion with medium-chain } \\
\text { TAG has long-lasting } \\
\text { cognition-enhancing } \\
\text { effects in aged dogs }\end{array}$ & PAN et al., & 2010 & $\begin{array}{l}\text { O presente estudo se con- } \\
\text { centrou na hipótese de que } \\
\text { a suplementação dietética } \\
\text { com TAG de cadeia média } \\
\text { (MCT) melhorará a função } \\
\text { cognitiva em cães idosos, } \\
\text { fornecendo energia ao cére- } \\
\text { bro na forma de cetonas. }\end{array}$ & $\begin{array}{l}\text { Esses resultados indicam, pri- } \\
\text { meiro, que a suplementação de } \\
\text { longo prazo com MCT pode ter } \\
\text { efeitos de melhoria da cognição } \\
\text { e, segundo que a suplementação } \\
\text { de MCT aumenta os níveis circu- } \\
\text { lantes de cetonas. Os resultados } \\
\text { suportam a hipótese de que a } \\
\text { função cerebral de cães idosos } \\
\text { pode ser melhorada pela suple- } \\
\text { mentação de MCT, que fornece } \\
\text { ao cérebro uma fonte de energia } \\
\text { alternativa. }\end{array}$ \\
\hline $\begin{array}{l}\text { Therapeutic agents for } \\
\text { the treatment of cog- } \\
\text { nitive dysfunction syn- } \\
\text { drome in senior dogs }\end{array}$ & $\begin{array}{l}\text { L A N D S - } \\
\text { BERG }\end{array}$ & 2005 & $\begin{array}{l}\text { Examinar os produtos que } \\
\text { agora são comercializados } \\
\text { junto com alguns que podem } \\
\text { ser considerados para uso } \\
\text { em cães idosos com disfun- } \\
\text { ção cognitiva, bem como a } \\
\text { pesquisa que tem sido usa- } \\
\text { da para validar a eficácia (ou } \\
\text { falta dela) desses compos- } \\
\text { tos. }\end{array}$ & $\begin{array}{l}\text { O modelo laboratorial canino para } \\
\text { avaliação do envelhecimento ce- } \\
\text { rebral provou ser valioso tanto } \\
\text { em estudos comparativos para a } \\
\text { doença de Alzheimer em huma- } \\
\text { nos quanto na compreensão do } \\
\text { diagnóstico e tratamento do en- } \\
\text { velhecimento cerebral em cães } \\
\text { idosos. }\end{array}$ \\
\hline $\begin{array}{l}\text { Canine cognitive dys- } \\
\text { function syndrome: Prev- } \\
\text { alence, clinical signs and } \\
\text { treatment with a neuro- } \\
\text { protective nutraceutical }\end{array}$ & \begin{tabular}{|l|} 
OSELLA \\
al.,
\end{tabular} & 2007 & 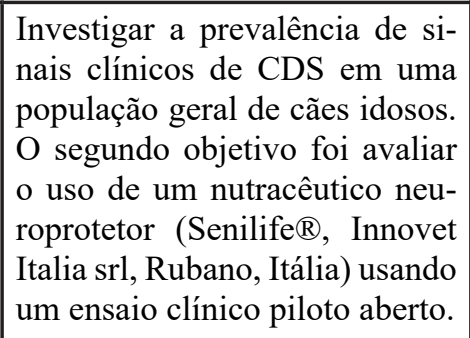 & $\begin{array}{l}\text { Os resultados preliminares dos cães } \\
\text { tratados com Senilife } ₫ \text { mostraram } \\
\text { uma melhora acentuada dos sinais } \\
\text { relacionados à CDS, mesmo que } \\
\text { os cães não tenham apresentado } \\
\text { remissão completa dos sintomas. }\end{array}$ \\
\hline $\begin{array}{l}\text { Nutritional supplemen- } \\
\text { tation in cases of canine } \\
\text { cognitive dysfunction }-\mathrm{A} \\
\text { Clinical Trial }\end{array}$ & $\begin{array}{l}\text { H E A T H ; } \\
\text { BARAB AS; } \\
\text { CRAZE. }\end{array}$ & 2007 & $\begin{array}{l}\text { Investigar os efeitos terapêuti- } \\
\text { cos de um suplemento especí- } \\
\text { fico quando comparado a um } \\
\text { placebo. }\end{array}$ & $\begin{array}{l}\text { Os resultados apoiam a prática clínica } \\
\text { da suplementação nutricional como } \\
\text { um valioso componente da aborda- } \\
\text { gem terapêutica em casos de disfun- } \\
\text { ção cognitiva canina. }\end{array}$ \\
\hline $\begin{array}{l}\mathrm{O} \text { enriquecimento am- } \\
\text { biental no bem-estar de } \\
\text { cães e gatos. }\end{array}$ & 00HENZEL & 2014 & $\begin{array}{l}\text { Apresentar uma revisão biblio- } \\
\text { gráfica da história, conceitua- } \\
\text { ção, e uso atual das técnicas de } \\
\text { enriquecimento ambiental em } \\
\text { ambientes habitados por cães e } \\
\text { gatos }\end{array}$ & $\begin{array}{l}\text { O enriquecimento é a chave para evi- } \\
\text { tar problemas comportamentais, per- } \\
\text { mitindo uma convivência saudável } \\
\text { entre tutor e animal. }\end{array}$ \\
\hline $\begin{array}{l}\text { Environmental enrich- } \\
\text { ment, new neurons and } \\
\text { the neurobiology of indi- } \\
\text { viduality }\end{array}$ & $\begin{array}{l}\text { K E M P E R - } \\
\text { MANN }\end{array}$ & 2019 & $\begin{array}{l}\text { Descrever oportunidades futu- } \\
\text { ras para o paradigma ENR que } \\
\text { residem em mudar o foco de } \\
\text { "enriquecimento da heredita- } \\
\text { riedade" para "enriquecimento } \\
\text { da plasticidade" e, assim, de } \\
\text { diferenças de ponto final está- } \\
\text { tico para efeitos em processos } \\
\text { dinâmicos. }\end{array}$ & $\begin{array}{l}\text { O paradigma ENR ajudou a moldar } \\
\text { a visão neurobiológica atual de que a } \\
\text { estrutura fina e a conectividade do cé- } \\
\text { rebro são influenciadas por nossa ex- } \\
\text { periência e - por mais que estejamos } \\
\text { influenciando o que experimentamos } \\
\text { e como reagimos a isso - nossa ati- } \\
\text { vidade no mundo. }\end{array}$ \\
\hline
\end{tabular}




\begin{tabular}{|c|c|c|c|c|}
\hline $\begin{array}{l}\text { Learning ability in aged } \\
\text { beagle dogs is preserved } \\
\text { by behavioral enrichment } \\
\text { and dietary fortification: } \\
\text { a two-year longitudinal } \\
\text { study }\end{array}$ & $\begin{array}{l}\text { MILGRAM, } \\
\text { et al., }\end{array}$ & 2005 & $\begin{array}{l}\text { Estender o modelo canino de } \\
\text { envelhecimento cognitivo com } \\
\text { uma investigação longitudinal } \\
\text { de dois anos de discriminação } \\
\text { e capacidade de aprendizagem } \\
\text { reversa em grupos de cães bea- } \\
\text { gle jovens e idosos. Com dois } \\
\text { objetivos, o primeiro foi obter } \\
\text { dados longitudinais de declínio } \\
\text { dependente da idade na capa- } \\
\text { cidade de aprendizagem. A se- } \\
\text { gunda foi avaliar a eficácia de } \\
\text { duas intervenções no combate } \\
\text { ao declínio dependente da ida- } \\
\text { de, enriquecimento comporta- } \\
\text { mental e uma alimento de ma- } \\
\text { nutenção fortificado com um } \\
\text { amplo espectro de antioxidan- } \\
\text { tes e cofatores mitocondriais }\end{array}$ & $\begin{array}{l}\text { Os resultados sugerem que o enrique- } \\
\text { cimento comportamental ou a fortifi- } \\
\text { cação da dieta com antioxidantes de } \\
\text { longa duração podem retardar o de- } \\
\text { clínio cognitivo dependente da idade, } \\
\text { e que os dois tratamentos juntos são } \\
\text { mais eficazes do que os dois sozinhos } \\
\text { em cães mais velhos. }\end{array}$ \\
\hline
\end{tabular}

\subsection{Manejo Nutricional}

O manejo nutricional tem função de suprir as necessidades fisiológicas dos animais e pode ser feito de diversas maneiras, por exemplo, com a utilização de alimentação natural, ração comercial, sendo essa a opção mais prática para o tutor, e com uso de suplementação.

Alguns nutrientes básicos devem estar presentes na dieta de cães acometidos com DCC, sendo o principal deles os antioxidantes. Por se tratar de uma doença onde ocorre uma maior deposição de placas beta-amiloide, que são neurotóxicas, diminuem a função mitocondrial, radicais livres de oxigênio e prejudicam a sinapse neuronal, é necessário fazer uso de antioxidantes, que podem trazer bons resultados na memória, aprendizagem e imunidade (BOS$\mathrm{CH}$ et al, 2012), pois eles têm diversas funções, dentre elas a capacidade de reduzir a produção de espécies reativas de oxigênio, diminuir o dano celular, tem poder anti-inflamatório, pode reduzir o acúmulo de placas beta-amiloides (PEREIRA, 2016), e também fazer reparação das lesões pelos radicais livres no DNA que podem ser diminuídas pela adição de frutas e vegetais na dieta (BIANCHI e ANTUNES, 1999).

Outras substâncias que devem ser citadas são a vitamina $E$, que impede os danos causados pelo envelhecimento, vitamina $\mathrm{C}$, betacaroteno e flavonoides, estes que conseguem sequestrar os radicais livres, selênio, glutationa, clorofilina e a L-cisteína (BIANCHI e ANTUNES, 1999).
As vitaminas $\mathrm{C}$ e $\mathrm{E}$ protegem contra $\mathrm{O}$ dano oxidativo no DNA mitocondrial pela idade e a oxidação da glutationa mitocondrial. A N-Acetil Cisteína, que é um precursor da glutationa e um antioxidante solúvel em água. A Co-Enzima Q-10 é um antioxidante solúvel em lipídios, que regenera a Vitamina $E$ e tem ação antioxidante, além de ser um estimulante de células $T$ e ter propriedades antitumorais. E para finalizar podemos citar também o Selênio e o ácido lipoico (HEATH; BARABAS; CRAZE, 2007).

Os cofatores mitocondriais como o ácido alfa lipóico e L-carnitina melhoram a eficiência da mitocôndria, diminuem os danos neurológicos, biológicos e também reduzem a produção dos radicais livres e aumentam o desenvolvimento muscular. Triglicerídeos de cadeia média acabam sendo uma segunda fonte de energia cerebral e reduzem os níveis de proteína percursora do amiloide. Ômegas 3 e 6 que atuam nas membranas cerebrais e ajudam na sua função normal. Ginko biloba reduz o dano oxidativo, inflamação, promove vasodilação e melhora a eficiência da mitocôndria (DRUCE, 2014; PEREIRA, 2016).

Dentre os alimentos que podem ser adicionados a alimentação natural, que não são tóxicos para os cães, e que contém antioxidantes e vitaminas podemos citar mamão, cenoura, brócolis, espinafre, batata-doce e peixe.

Partindo para outro ponto, em relação a rações comerciais, as neurológicas são uma opção para se fornecer a caninos acometidos com a DCC, porém infelizmente no Brasil só é 
possível encontrar a Pro Plan® Veterinary Diets NC Neurologic Care - Cuidado Neurológico que conta com alguns suplementos básicos para a doença como antioxidantes, vitaminas $\mathrm{E}, \mathrm{C}$ e $B$, piridoxina, zinco e manganês (KRUG, 2018; PRO PLAN, 2021).

Segundo Pan (2010), os animais alimentados com essa ração melhoram o declínio cognitivo relacionado a idade por fornecer uma fonte alternativa de energia para o cérebro. Outra opção que está disponível apenas nos EUA e foi formulada justamente para ajudar na memória e aprendizado de cães idosos é a ração da Hills, a b/d Dry Dog Food que fortalece o cérebro contra os efeitos cognitivos trazidos pelo envelhecimento e contém antioxidantes, vitaminas e minerais (HILLS, 2021; KRUG, 2018; LANDSBERG, 2005).

Um ensaio clínico realizado por Osella et al., (2007) utilizou um suplemento alimentar que é usado nos Estados Unidos o Senilife, que inclui alguns componentes que ajudam no funcionamento cerebral, como a fosfatidilserina que melhora a comunicação neural, piridoxina que é essencial para o desenvolvimento e saúde do cérebro, o extrato de Gingko biloba aumenta o metabolismo da glicose, fluxo sanguíneo cerebral e tem efeito antioxidante, resveratrol tem ação antioxidante e o D-alfa-tocoferol é uma forma natural da vitamina $E$ que é antioxidante, e que mostrou uma diminuição na severidade dos sintomas.

A Fosfatidilserina facilita o transporte de impulso elétrico dos neurônios e foi comprovado que melhora na recuperação da memória e o comportamento exploratório (HEATH; BARABAS; CRAZE, 2007).

Um estudo feito por Heath, Barabas e Craze (2007) com o Aktivait, outro suplemento vendido nos EUA, mas que está disponível para entrega no Brasil, fornecido a 20 cães com Disfunção Cognitiva Canina mostrou que se obteve uma diminuição na quantidade de horas dormidas durante o dia e melhora no reconhecimento do tutor e interação social.

O suplemento é dividido em 4 grupos. Primeiro são os ácidos graxos, a DHA/EPA e o fosfolipídio Fosfatidilserina que são fortalecedores cerebrais. $\mathrm{O}$ segundo e terceiro grupo são os que causam o aumento de sinalização e aumento metabólico, e eles são o Acetil-L- Carnitina e L-Carnitina que são responsáveis por carregar ácidos graxos para células, e a combinação deles em animais idosos pode prevenir o decaimento mitocondrial em neurônios e restaurar a função cognitiva. $E$ o quarto e último grupo, é composto pelos antioxidantes (HEATH; BARABAS; CRAZE, 2007).

Percebe-se que há inúmeros alimentos, além de outras opções que podem propiciar aos caninos melhora na qualidade de vida, visto que é de essencial importância o acompanhamento e o conhecimento da dieta a ser fornecida. Dessa forma com o devido cuidado os índices de DCC podem diminuir a de certa forma amenizar nos cães já acometidos.

\subsection{Enriquecimento Ambiental}

O enriquecimento ambiental é uma ciência que busca melhorar a qualidade de vida dos animais por meio de atividades e estímulos que os incentivam a interagir e assim aumentar seu bem-estar físico e mental. Pode ser dividido em cinco categorias apresentadas a seguir: alimentar, cujo objetivo é oferecer o alimento em formatos e texturas diferentes, além de variação na frequência e no horário de fornecimento, o sensorial que incentiva os sentidos através de cheiros e sons, o físico através das mudanças nos cômodos, o cognitivo para estimular mentalmente com resolução de problemas e o social que comporta a interação entre mesma espécie ou espécies diferentes (HENZEL, 2014).

A utilização dessa estratégia juntamente com uma dieta adequada ajuda a diminuir os sintomas apresentados por animais com DCC. Já foi constatado que o EA induz a formação de novas sinapses e ramificação dendrítica e essas mudanças anatômicas ajudam na memória e se consolidam dependendo do tempo de estímulo, ou seja, quanto mais cedo e por mais tempo esses estímulos forem aplicados, melhores serão essas mudanças no cérebro (FISHER, 2015).

Animais que passaram a receber o enriquecimento, apresentaram aumento da recuperação de condições agudas, alteração na expressão gênica de células e tecidos, produção de proteínas, processos bioquímicos de neurotransmissores, hormônios e fatores imu- 
nológicos que duram mesmo após o cessar do estímulo, sendo importante que o EA seja composto de atividade física, interação social e estimulação cognitiva (KEMPERMANN, 2019).

O enriquecimento cognitivo pode ser aplicado de diversas formas, seja ao praticar truques previamente aprendidos ou mesmo ensinar novos, como o de sentar-se e ficar, com o recebimento de recompensa a cada acerto, revezamento de brinquedos semanalmente (MILGRAM et al., 2005), e ao realizar passeios rotineiramente explorando caminhos diferentes que ajudam a estimular a interação social com outros animais e pessoas, que é também um tipo de enriquecimento social (PEREIRA, 2016).

Para estimular a memória e aprendizagem dos animais, algumas brincadeiras e melhorias no ambiente podem ser feitas. Por exemplo, para introduzir um enriquecimento ambiental alimentar, sensorial e cognitivo podemos esconder um petisco ou fruta em algum local da casa e estimular o cão a utilizar o olfato para procurar, esconder dentro de uma caixa a fim de fazer o animal desenvolver a habilidade de solucionar problemas para que consiga liberar a comida, e a utilização de velas em certos cômodos da casa, para ajudar o animal a reconhecer o cômodo (COUPLAND; REYNOLDS, 2018; DRUCE, 2014; HENZEL, 2014).

Em relação ao enriquecimento físico, algumas mudanças no local onde o cão vive devem ser feitas de acordo com a sua necessidade. Caso ele tenha dificuldade de locomoção pelas características do piso, a colocação de tapetes antiderrapantes pode ser uma solução; distribuir diversas vasilhas de água pela casa estimula a ingestão hídrica e evita que o animal tenha que se descolar muito para se hidratar (PEREIRA, 2016). Um cuidado que se dever ter é fazer o enriquecimento no ambiente gradualmente, pois cães com DCC muitas vezes ficam estressados após mudanças na sua rotina.

Desta maneira o protocolo de tratamento de cães com a disfunção cognitiva canina deve incluir sempre a junção do manejo nutricional com os nutrientes necessários para reduzir a deposição das placas beta-amiloioide através da ração comercial ou alimentação natural, e da introdução do enriquecimento ambiental.

\section{CONCLUSÃO}

A disfunção cognitiva canina é uma doença comportamental que acomete cães de qualquer raça e sexo, geralmente com mais de oito anos de idade. O diagnóstico é feito a base dos sinais clínicos apresentados e da aplicação de um pequeno questionário aos tutores com o intuito de se reconhecer os sintomas, e o tratamento é feito de 3 maneiras, o farmacológico, nutricional e de enriquecimento do ambiente.

$O$ uso de antioxidantes e vitaminas neuroprotetoras como vitamina $\mathrm{E}$ e $\mathrm{C}$, e a L-carnitina na dieta é de suma importância pois trazem bons resultados na memória, aprendizagem e imunidade, já que são capazes de diminuir o dano celular, tem poder anti-inflamatório e pode reduzir o acúmulo de placas beta-amiloides. 0 enriquecimento ambiental tem que ser composto de atividade física através de passeios diários por caminhos diferentes, interação social com outros animais e pessoas e estimulação cognitiva por brincadeiras de esconder alimentos com o objetivo de fazer o cão usar o olfato para buscar e desenvolver habilidade de solucionar o problema.

Á vista disso a mudança alimentar juntamente com o uso do enriquecimento ambiental são alternativas para desacelerar o avanço da DCC e aumentar a qualidade de vida do cão, assim como sua interação com o tutor, mas temos que manter em mente que por se tratar de uma doença neurodegenerativa ainda não existe cura, e sim um tratamento paliativo e que ajuda a regredir os sintomas apresentados pelos animais.

\section{REFERÊNCIAS}

AZKONA, G. et al. Prevalence and risk factors of behavioural changes associated with age-related cognitive impairment in geriatric dogs. J Small Anim Pract, [s. I], v. 50, n. 2, p. 87-91, 04 fev. 2009. Disponível em: 10.1111/j. 1748-5827.2008.00718.x. Acesso em: 05 jun. 2021

BIANCHI, Maria de Lourdes Pires e ANTUNES, Lusânia Maria Greggi Radicais livres e os principais antioxidantes da dieta. Revista de 
Nutrição [online]. 1999, v. 12, n. 2 [Acessado 3 Julho2021], pp. 123-130. Disponívelem:<https:// doi.org/10.1590/S1415-52731999000200001>. Epub 07 Out 2004. ISSN 1678-9865. https://doi. org/10.1590/S1415-52731999000200001.

BOSCH, Maria Neus; PUGLIESE, Marco; GIMENO-BAYÓN, Javier; RODRÍGUEZ, Manuel José; MAHY, Nicole. Dogs with cognitive dysfunction syndrome: a natural model of Alzheimer's disease. Curr Alzheimer Res, [s. ]], v. 3, p. 298-314, 2012. Disponível em: doi:10.2174/156720512800107546. Acesso em: 05 jun. 2021.

COTMAN CW, Head E, MUGGENBURG BA, ZICKER S, MILGRAM NW. Brain aging in the canine: a diet enriched in antioxidants reduces cognitive dysfunction. Neurobiol Aging. 2002 Sep-Oct;23(5):809-18. doi: 10.1016/ s0197-4580(02)00073-8. PMID: 12392784.

COUPLAND, S., \& REYNOLDS, H. Do dog owners recognise behavioural indicators of canine cognitive dysfunction and can environmental enrichment techniques slow its progression?. The Veterinary Nurse, 2018. 9(2), 118-123. doi:10.12968/vetn.2018.9.2.118

DRUCE, Kelly. Canine cognitive dysfunction - recognition and treatment. Veterinary Nursing Journal, [s. I], v. 29, n. 8, p. 268-270, 28 jan. 2015. Disponível em: https:// doi.org/10.1111/vnj.12164. Acesso em: 06 jun. 2021.

FISCHER A. Environmental enrichment as a method to improve cognitive function. What can we learn from animal models? Neuroimage. 2016 May 1;131:42-7. doi: 10.1016/j. neuroimage.2015.11.039. Epub 2015 Nov 30. PMID: 26656208.

HEATH, S. E., BARABAS, S., \& CRAZE, P. G. Nutritional supplementation in cases of canine cognitive dysfunction-A clinical trial. Applied Animal Behaviour Science, 2007. 105(4), 284-296. doi:10.1016/j.applanim.2006.11.008
HENZEL, Marcelo. 0 enriquecimento ambiental no bem-estar de cães e gatos. 53 f. Monografia (Especialização) - Curso de Medicina Veterinária, Universidade Federal do Rio Grande do Sul, Porto Alegre, 2014.

\section{HILLS Prescription Diet b/d Dry Dog}

Food. 2021. Disponível em: https://www.hillspet.com/dog-food/pd-bd-canine-dry. Acesso em: 29 maio 2021.

KEMPERMANN, G. Environmental enrichment, new neurons and the neurobiology of individuality. Nat Rev Neurosci. 2019.20, 235-245. https://doi.org/10.1038/s41583-019$0120-x$

KRUG, Fernanda Dagmar Martins et al. Síndrome de disfunção cognitiva canina. Revista Científica de Medicina Veterinária - Pequenos Animais e Animais de Estimação, Pelotas, v. 46, n. 15, p. 106-110, jul. 2016.

KRUG, F.D.M , et al. Avaliação diagnóstica na síndrome disfunção cognitiva canina. 2018. Arq. Bras. Med. Vet. Zootec., v.70, n.6, p.1723-1730

LANDSBERG G, ARAUJO JA. Behavior problems in geriatric pets. Vet Clin North Am Small Anim Pract. 2005 May;35(3):675-98. doi: 10.1016/j.cvsm.2004.12.008. PMID: 15833565.

LANDSBERG, G. Therapeutic agents for the treatment of cognitive dysfunction syndrome in senior dogs. Progress in Neuro-Psychopharmacology and Biological Psychiatry, 2005. 29(3), 471-479. doi:10.1016/j. pnpbp.2004.12.012

LIMA, M. T, d R "Efeitos da Acupuntura na Disfunção Cognitiva Canina" [Dissertação de pós-Graduação]. Rio de Janeiro: Universidade Federal do Rio de Janeiro; 2018

MILGRAM, N. W., HEAD, E., ZICKER, S. C., IKEDA-DOUGLAS, C. J., MURPHEY, H., MUGGENBURG, B., COTMAN, C. W. Learning ability in aged beagle dogs is preserved by behavioral enrichment and dietary fortification: 
a two-year longitudinal study. Neurobiology of Aging, 2005. 26(1), 77-90. doi:10.1016/j.neurobiolaging.2004.02.014

OSELLA, M. C., Re, G., ODORE, R., GIRARDI, C., BADINO, P., BARBERO, R., \& BERGAMASCO, L. Canine cognitive dysfunction syndrome: Prevalence, clinical signs and treatment with a neuroprotective nutraceutical. Applied Animal Behaviour Science, 2007 105(4), 297310. doi:10.1016/j.applanim.2006.11.007

PAN, Y., LARSON, B., ARAUJO, J. A., LAU, W., de RIVERA, C., SANTANA, R., MILGRAM, N. W. Dietary supplementation with medium-chain TAG has long-lasting cognition-enhancing effects in aged dogs. British Journal of Nutrition, 2010. 103(12), 17461754. doi: $10.1017 / \mathrm{s} 0007114510000097$

PEREIRA, R. M. da C. A Síndrome da Disfunção Cognitiva Canina. 2016. 44p Relatório Final de Estágio (Mestrado de Medicina Veterinária). Instituto de Ciências Biomédicas. Universidade do porto, Porto, 2016.

PRO Plan® Veterinary Diets NC Neurologic Care - Cuidado Neurológico - Fórmula Canina. 2021. Disponível em: https://www.nestle.com.br/marcas/purina-pro-plan/pro-plan-veterinary-diets-nc-neurologic-care-cuidado-neurologico-formula-canina. Acesso em: 29 maio 2021.

SEKSEL, K. Canine Cognitive Dysfunction. .2012. Disponível em http://dogdementia. com/ Acesso em 29 de Maio 2021

SILVA, Bruna Carvalho da; GNEIDING, Beatriz; LUCIOLI, Joelma; TESSER, Jéssica Sara; GNEIDING, José Eduardo Basilio de Oliveira. Síndrome da disfunção cognitiva canina: revisão de literatura. Revista Acadêmica Ciência Animal, [S.L.], v. 16, p. 1, 28 ago. 2018. Pontificia Universidade Catolica do Parana - PUCPR. http://dx.doi.org/10.7213/19814178.2018.161108.

SORDO, Lorena; GUNN-MOORE, Danièlle A.. Cognitive Dysfunction in Cats: update on neuropathological and behavioural changes plus clinical management. Veterinary Record, [S.L.], v. 188, n. 1, p. 1-12, jan. 2020. Wiley. http://dx.doi.org/10.1002/vetr.3. 